\title{
Real ideals of compact operators of complex factors
}

\author{
Sergio Albeverio • Rashidkhon A. Dadakhodjaev • \\ Abdugafur A. Rakhimov
}

Received: 3 November 2012 / Accepted: 21 January 2013 / Published online: 22 February 2013 (C) The Author(s) 2013. This article is published with open access at Springerlink.com

\begin{abstract}
Real ideals of compact operators for (complex) factors are investigated. A description (up to isomorphisms) of real two-sided ideals of relatively compact operators of a complex $\mathrm{W}^{*}$-factors is given. A relative weak $(\mathrm{RW})_{r}$ convergence in a real Hilbert space is introduced. The classical Hilbert characterization of compactness of operators is extended to the compact operators in semifinite real $\mathrm{W}^{*}$-algebras.
\end{abstract}

Keywords $\mathrm{W}^{*}$-algebras $\cdot$ Real factors $\cdot$ Ideals $\cdot$ Compact operators

Mathematics Subject Classification (2000) $\quad 46 \mathrm{~L} 10 \cdot 46 \mathrm{~L} 37$

S. Albeverio

Institut für Angewandte Mathematik, Universität Bonn, Endenicher Allee 60, 53115 Bonn, Germany e-mail: albeverio@uni-bonn.de

S. Albeverio

HCM, SFB 611 and IZKS, Bonn, Germany

S. Albeverio

BiBoS, Bielefeld, Germany

S. Albeverio

CERFIM, Locarno, Switzerland

R. A. Dadakhodjaev

Institute of Mathematics, National University of Uzbekistan,

Vuz gorodok, 100095 Tashkent, Uzbekistan

e-mail: dadakhodjaev@iam.uni-bonn.de

A. A. Rakhimov ( $\square)$

Tashkent Automobile and Road Construction Institute,

20 Amir Temir Shoh. Str., 100060 Tashkent, Uzbekistan

e-mail: gafur_rakhimov@yahoo.com

Birkhäuser 


\section{Introduction}

In the present paper we investigate the real ideals of compact operators for complex factors and give a description (up to isomorphisms) of real two-sided ideal of relatively compact operators of the complex $\mathrm{W}^{*}$-factors. A concept of relative weak $(\mathrm{RW})_{r}$ convergence in a real Hilbert space is introduced. The classical Hilbert characterization of compactness of operators is extended to the compact operators in semifinite real $\mathrm{W}^{*}$-algebras.

\section{Preliminaries}

Let $B(H)$ be the algebra of all bounded linear operators on a complex separable Hilbert space $H$. A weakly closed *-subalgebra $\mathfrak{A}$ containing the identity operator $\mathbf{I}$ in $B(H)$ is called a $\mathrm{W}^{*}$-algebra. A real *-subalgebra $R \subset B(H)$ is called a real $\mathrm{W}^{*}$-algebra if it is closed in the weak operator topology, $\mathbf{I} \in R$ and $R \cap i R=\{0\}$. A real W*-algebra $R$ is called a real factor if its center $Z(R)$ consists of the elements $\{\lambda \mathbf{I}, \lambda \in \mathbb{R}\}$, where $\mathbb{R}$ is the field of all real numbers. We say that a real $\mathrm{W}^{*}$-algebra $R$ is of the type $\mathrm{I}_{\text {fin }}, \mathrm{I}_{\infty}, \mathrm{II}_{1}$, $\mathrm{II}_{\infty}$, or $\mathrm{III}_{\lambda},(0 \leq \lambda \leq 1)$ if the enveloping $\mathrm{W}^{*}$-algebra $\mathfrak{A}(R)$ has the corresponding type in the ordinary classification of $\mathrm{W}^{*}$-algebras. A linear mapping $\alpha$ of an algebra into itself with $\alpha\left(x^{*}\right)=\alpha(x)^{*}$ is called an *automorphism if $\alpha(x y)=\alpha(x) \alpha(y)$; it is called an involutive *-antiautomorphism if $\alpha(x y)=\alpha(y) \alpha(x)$ and $\alpha^{2}(x)=x$. If $\alpha$ is an involutive *-antiautomorphism of a $\mathrm{W}^{*}$-algebra $M$, we denote by $(M, \alpha)$ the real $\mathrm{W}^{*}$-algebra generated by $\alpha$, i.e. $(M, \alpha)=\left\{x \in M: \alpha(x)=x^{*}\right\}$. Conversely, every real $\mathrm{W}^{*}$-algebra $R$ is of the form $(M, \alpha)$, where $M$ is the complex envelope of $R$ and $\alpha$ is an involutive *-antiautomorphism of $M$ (see [1,2,5,9]). Therefore we shall identify from now on the real von Neumann algebra $R$ with the pair $(M, \alpha)$.

A trace on a (complex or real) $\mathrm{W}^{*}$-algebra $N$ is a linear function $\tau$ on the set $N^{+}$ of positive elements of $N$ with values in $[0,+\infty]$, satisfying $\tau\left(u x u^{*}\right)=\tau(x)$, for an arbitrary unitary $u$ and for any $x$ in $N$.

The trace $\tau$ is said to be finite, if $\tau(\mathbf{I})<+\infty$; semifinite, if given any $x \in N^{+}$there is a nonzero $y \in N^{+}, y \leq x$ with $\tau(y)<+\infty$.

\section{Real and complex ideals of $W^{*}$-algebras}

Definition 1 Let $M$ be a $\mathrm{W}^{*}$-algebra. A real subspace $I$ of $M$ is called a real ideal of $M$ if $I \cdot M \subset I_{c}$, where $I_{c}$ is the smallest complex subspace of $M$, containing $I$.

It is easy to see that the subspace $I_{C}$ is equal to $I+i I$, therefore a real subspace $I$ is a real ideal if and only if $I \cdot M \subset I+i I$.

Since each complex subspace of $M$ is a real subspace, any complex ideal is automatically a real ideal of $M$. Let $I$ be a real ideal of $M$. If there exists a real $\mathrm{W}^{*}$-subalgebra $R$ of $M$ with $R+i R=M$, such that $I \subset R$, then $I$ is called a pure real ideal of $M$. In this case, it is obvious that we have $I \cdot R \subset I$. Note that, the reverse is not true, i.e. from $I \cdot R \subset I$ it does not follow $I \subset R$. But a complex subspace $J=I+i I$ always is a complex ideal of $M$. On the other hand if $I \subset R$ is a real subspace of $M$ and $I+i I$ is a complex ideal, then $I$ is a pure real ideal, i.e. we obtain $I \cdot R \subset I$. 
Let, now $I$ and $Q$ be pure real ideals of $M$. In general, the set $I+i Q$ is not a (complex) subspace. More precisely the set $I+i Q$ is a complex subspace if and only if $I=Q$. Therefore we consider the smallest complex subspace $J$ of $M$, containing $I$ and $Q$. Obviously $J$ is equal to $(I+Q)+i(I+Q)$. Thus, if $I$ and $Q$ are real ideals, then $J=(I+Q)+i(I+Q)$ is a complex ideal.

\section{Ideals of compact operators}

Let $(M, \alpha)$ be a real factor and let $\tau$ be an $\alpha$-invariant semifinite trace on $M$. A subspace $K \subset H$ is called $\tau$-finite (or finite relative to $\tau$ ), if $\tau\left(P_{K}\right)<+\infty$, where $P_{K}$ is the canonical projection of $H$ on $K$ with $P_{K} \in M$.

Now, let $K$ be a subset of $H$. A subset $K$ is called $\tau$-compact (or compact relative to $\tau$ ), if $\mathrm{K}$ is approximated in the norm $\|\cdot\|_{H}$ by a bounded sequence of $\tau$-finite subspaces.

A real operator $A$ on $H$ (i.e. $A \in(M, \alpha))$ is called real compact relative to $\tau$ if it is an operator mapping bounded sets into relatively compact sets. We denote by $I$ (respectively, by $J$ ) the set of all relatively compact operators of $(M, \alpha)$ (respectively, of $M$ ). Let us recall the following result.

Theorem 1 ([3,10,11]) Let $M$ be a semifinite factor and let $\alpha$ be an involutive *-antiautomorphism of $M$. Then I (respectively, $J$ ) is a unique (nonzero) uniformly closed two-sided ideal of $(M, \alpha)$ (respectively, of $M$ ), and $I+i I=J$.

Now, let us recall [4] the notion of the crossed product of a $\mathrm{W}^{*}$-algebra $M$ by a locally compact topological group $G$ by its *-automorphism. Let $\gamma: G \rightarrow \operatorname{Aut}(M)$ be a group homomorphism such that the map $g \rightarrow \gamma_{g}$ is strongly continuous. Let $L_{2}(G, H)$ be the Hilbert space of all $H$-valued square integrable functions on $G$. We consider a *-algebra $N \subset B\left(L_{2}(G, H)\right)$, generated by operators of the form: $\pi_{\gamma}(a)$ and $u(g)$, where $a \in M, g \in G$, and

$$
\left(\pi_{\gamma}(a) \xi\right)=\gamma_{h}^{-1}(a) \xi(h), \quad(u(g) \xi)(h)=\xi\left(g^{-1} h\right)
$$

$\xi=\xi(h) \in L_{2}(G, H), g, h \in G$. The algebra $N$ is called the crossed product of $M$ by $G$, and denoted by $W^{*}(M, G)$ or $M \times{ }_{\gamma} G$. Moreover, there exists a canonical embedding $\pi \gamma: M \rightarrow \pi_{\gamma}(M) \subset N$. Each element $x \in N$ has the form: $x=\sum_{g \in G} \pi_{\gamma}(x(g)) u(g)$, where $x(\cdot)$ is an $M$-valued function on $G$. If $\theta$ is a *-automorphism of $M$, then for the action $\left\{\theta^{n}\right\}$ of the group $\mathbb{Z}$ on $M$ we denote by $W^{*}(\theta, M)$ or $M \times_{\theta} \mathbb{Z}$ the crossed product of $M$ by $\theta$. Similarly one can define the notion of crossed product for real $\mathrm{W}^{*}$-algebras (see [1,12-14]).

Let $M$ be a factor of type $\operatorname{III}_{\lambda}(\lambda \neq 1)$ and let $\alpha$ be an involutive *-antiautomorphism of $M$. Then by [12] (see also [1]), either

- there exist a factor $N$ of type $\mathrm{II}_{\infty}$ and an $\alpha$-invariant automorphism $\theta$ of $N$ such that $(M, \alpha)$ is isomorphic to the (real) crossed product $(N, \alpha) \times_{\theta} \mathbb{Z}$ or

- there exist a factor $N$ of type $\mathrm{II}_{\infty}$ and an antiautomorphism $\sigma$ of $N$ such that $(M, \alpha)$ is isomorphic to $\left(\left(N \oplus N^{o p}\right) \times_{\sigma} \mathbb{Z}, \beta\right)$, where $N^{o p}$ is the opposite $\mathrm{W}^{*}$-algebra for $N$ and $\beta(x, y)=(y, x)$, for all $x, y \in N$. 
Let's consider the first case. Let $(M, \alpha)$ be isomorphic to the (real) crossed product $(N, \alpha) \times_{\theta} \mathbb{Z}$. It is known that $(N, \alpha) \times_{\theta} \mathbb{Z}+i \cdot(N, \alpha) \times_{\theta} \mathbb{Z}=N \times_{\theta} \mathbb{Z}$ (see [12-14]). Let $E: M \rightarrow N$ be the unique $\alpha$-invariant faithful normal conditional expectation (see $[15,16])$. Let us state an auxiliary lemma whose proof immediately follows from the linearity and $\alpha$-invariance of $E$.

Lemma 1 If $S$ is an ideal in $(M, \alpha)$ and $S_{c}=S+i S$, then

$$
E^{-1}(S)+i E^{-1}(S)=E^{-1}\left(S_{c}\right), \quad E^{-1}(S)^{+} \subset E^{-1}(S)^{+},
$$

where $E^{-1}(A)=\{x: E(x) \in A\}$.

Recall that a cone $K \subset A^{+}$is called hereditary if $x \in A^{+}, y \in K$ and $x \leq y$ implies $x \in K$; a subalgebra $B \subset A$ is called hereditary if the cone $B^{+}$is hereditary. It is easy to see that any two-sided ideal is hereditary.

Lemma 2 If the cone $E^{-1}\left(S_{c}\right)^{+}$is hereditary, then the cone $E^{-1}(S)^{+}$is also hereditary.

Proof If $x \in(M, \alpha)^{+} \subset M^{+}, y \in E^{-1}(S)^{+} \subset E^{-1}\left(S_{c}\right)^{+}$and $x \leq y$, then $x \in E^{-1}\left(S_{c}\right)^{+}$, since $E^{-1}\left(S_{c}\right)^{+}$is hereditary. Therefore $\alpha(x)=x^{*}$ implies $x \in E^{-1}(S)^{+}$.

Proposition 1 Let $M$ be a semifinite factor. If $S \subset(M, \alpha)$ is a two-sided ideal, then the linear span of $E^{-1}(S)^{+}$denoted as span $\left(E^{-1}(S)^{+}\right)$is a hereditary $*_{\text {-subalgebra }}$ of $(M, \alpha)$ and a two-sided module over $(M, \alpha)$. Moreover, if $S$ is a norm-closed, then $\operatorname{span}\left(E^{-1}(S)^{+}\right)$is also norm-closed.

Proof By Lemma 1 and Proposition 3.3 [6] the cone $E^{-1}\left(S_{c}\right)^{+}$is hereditary and $\operatorname{span}\left(E^{-1}\left(S_{c}\right)^{+}\right)$is a hereditary *-subalgebra of $M$, where $S_{c}=S+i S$. By Lemma 2 the cone $E^{-1}(S)^{+}$is also hereditary. Using the hereditarity of $\operatorname{span}\left(E^{-1}\left(S_{c}\right)^{+}\right)$one can easily check the hereditarity of $\operatorname{span}\left(E^{-1}(S)^{+}\right)$.

If $S$ is norm-closed, then $S^{+}$is also norm-closed. The continuity of $E$ implies that $E^{-1}(S)^{+}$is closed. Therefore $\operatorname{span}\left(E^{-1}(S)^{+}\right)$is also closed.

Let $x \in E^{-1}(S)^{+}$and $y \in(M, \alpha)$. From $x \in E^{-1}\left(S_{c}\right)^{+}$and $y \in M$, by Proposition $3.3[6]$ we obtain that $y x \in \operatorname{span}\left(E^{-1}\left(S_{c}\right)^{+}\right)$, since $\operatorname{span}\left(E^{-1}\left(S_{c}\right)^{+}\right)$ is a two-sided module over $M$. On the other hand $y x \in(M, \alpha)$ and $\{a \in$ $\left.\operatorname{span}\left(E^{-1}\left(S_{c}\right)^{+}\right): \alpha(a)=a^{*}\right\}=\operatorname{span}\left(E^{-1}(S)^{+}\right)$. Hence $y x \in \operatorname{span}\left(E^{-1}(S)^{+}\right)$. Since the element $x$ is arbitrary from $E^{-1}(S)^{+}$by linearity we obtain $y x \in$ $\operatorname{span}\left(E^{-1}(S)^{+}\right)$for any $x \in \operatorname{span}\left(E^{-1}(S)^{+}\right)$. Therefore $\operatorname{span}\left(E^{-1}(S)^{+}\right)$is a leftsided module over $(M, \alpha)$. Similarly one can show, that it is a right-sided $(M, \alpha)$ module.

Now, we put $\mathcal{I}=\operatorname{span}\{x \in(M, \alpha): E(x) \in I\}$, where $I$ is the unique (nonzero) uniformly closed two-sided ideal of the semifinite real factor $(N, \alpha)$ (see Theorem 1$)$. By Proposition $1, \mathcal{I}$ is hereditary.

Lemma 3 The following is valid

$$
\mathcal{I}^{+}=\left\{x \in(M, \alpha)^{+}: x \leq y, \quad \text { for some } y \in I^{+}\right\} .
$$

Proof Let $x \in \mathcal{I}^{+}$and $J=I+i I, \mathcal{J}^{+}=\left\{x \in M^{+}: x \leq z\right.$, for some $\left.z \in J^{+}\right\}$. Since $x \in \mathcal{J}^{+}$by Proposition 3.7 d) [6], $x \leq z$, for some $z \in J^{+}$. Let $z=y+i t$, 
$y, t \in(N, \alpha)$. Then $y \geq 0$ (because $z \geq 0)$ and $z-x=(y-x)+i t \geq 0$, hence $y-x \geq 0$, i.e., $x \leq y$. Since $I^{+} \subset J^{+}, y \in I^{+}$.

Conversely, if $x \in(M, \alpha)^{+}$and $x \leq y$, for some $y \in I^{+}$, then again by Proposition $3.7 \mathrm{~d})$ [6] we have $x \in \mathcal{J}^{+}$. From $\alpha(x)=x^{*}$ we have $x \in \mathcal{I}^{+}$.

From Lemma 3 , in particular, it follows, that a projection from $(M, \alpha)$ is finite if and only if it majorized by some finite projection of $(N, \alpha)$.

Let $I_{1}$ be the norm closure of $\mathcal{I}$. If we apply Proposition 1, Lemma 3 and the scheme of the proofs of Propositions 4.1, 4.5 and Theorem 4.3 [6], then we can prove the following real analogue of Halpern-Kaftal's theorem.

Theorem 2 Let $M$ be a factor of type $\operatorname{III}_{\lambda}(\lambda \neq 1)$ and let $\alpha$ be an involutive *-antiautomorphism of $M$. If the real factor $(M, \alpha)$ is isomorphic to the (real) crossed product $(N, \alpha) \times_{\theta} \mathbb{Z}$, then $I_{1}$ is a unique (up to an inner automorphism) smallest hereditary real $C^{*}$-subalgebra of $(M, \alpha)$, containing the ideal $I$, and it is a two-sided module over $(N, \alpha)$.

Let us consider the second case. Let $(M, \alpha)$ be isomorphic to $\left(\left(N \oplus N^{o p}\right) \times_{\sigma} \mathbb{Z}, \beta\right)$, where $N$ is a $\mathrm{II}_{\infty}$-factor, $N^{o p}$ is the opposite $\mathrm{W}^{*}$-algebra for $N$ and $\beta(x, y)=(y, x)$, for all $x, y \in N$.

Recall that, a factor $N$ is generated by the fixed point algebra of the one parameter group $\left\{\sigma_{t}^{\psi}: t \in \mathbb{R}\right\}$ of modular automorphisms, associated with some $\alpha$-invariant faithful normal semifinite weight $\psi$. More precisely, the $\mathrm{W}^{*}$-subalgebra $M_{\psi}=\{x \in$ $\left.M: \sigma_{t}^{\psi}(x)=x, t \in \mathbb{R}\right\}$ contains a central projection $p$ such that $N$ is isomorphic to factor $p M_{\psi}$. In this case the real $\mathrm{W}^{*}$-algebra $\left(M_{\psi}, \alpha\right)$ is isomorphic to $\left(N \oplus N^{o p}, \beta\right)$ (for more details see [1,12-14]).

Let $E:(M, \alpha) \rightarrow\left(N \oplus N^{o p}, \beta\right)$ be a faithful normal conditional expectation (see $[15,16]$ ) and let $J$ be the unique (nonzero) uniformly closed two-sided ideal of the semifinite (complex) factor $N$ (see Theorem 1). Similarly to the first case, we denote by $\mathrm{I}_{2}$ the norm closure of $\operatorname{span}\left\{x \in(M, \alpha): E(x) \in\left(J \oplus J^{o p}, \beta\right)\right\}$.

Applying the same reasonings, as in the first case, and the scheme of proofs of Propositions 4.1, 4.5 and Theorem 4.3 [6], we obtain one more real analogue of Halpern-Kaftal's theorem.

Theorem 3 Let $M$ be a factor of type $\operatorname{III}_{\lambda}(\lambda \neq 1)$ and let $\alpha$ be an involutive *-antiautomorphism of $M$. If the real factor $(M, \alpha)$ is isomorphic to $\left(\left(N \oplus N^{o p}\right) \times_{\sigma} \mathbb{Z}, \beta\right)$, then $I_{2}$ is the unique (up to inner automorphism) smallest hereditary real $C^{*}$-subalgebra of $(M, \alpha)$, containing the ideal I and is a two-sided module over $(N, \alpha)$. Here I is the unique (nonzero) uniformly closed two-sided ideal of semifinite real factor $(N, \alpha)$.

Thus, summarizing all above, in the injective case, we can describe all (nonzero) uniformly closed two-sided real ideals of semifinite and pure infinite complex factors. Recall that [1,5], if $M$ is an injective factor of type II, then there exists a unique conjugacy class of involutive *-antiautomorphisms in $M$; therefore there exists a unique (up to isomorphisms) real subfactor of $M$, generating $M$. If $M$ is an injective factor of type $\mathrm{III}_{\lambda}(0<\lambda<1)$, then in $M$ there exist exactly two conjugacy classes of involutive *-antiautomorphism; therefore there exist two (up to isomorphisms) real subfactors of $M$, generating $M$. Hence we obtain the following result. 
Theorem 4 Let $M$ be a factor. Then the following assertions are true:

1. If $M$ is an injective factor of type $I I_{1}$ or type $I_{\infty}$, then there exist (up to isomorphisms) two (nonzero) uniformly closed two-sided real ideals in $M$. One of them is the complex ideal $J$, the other is the pure real ideal $I$.

2. If $M$ is an injective factor of type $\operatorname{III}_{\lambda}(0<\lambda<1)$, then there exist (up to isomorphisms) three (nonzero) uniformly closed two-sided real ideals in M. One of them is the complex ideal $J$, the two others are the pure real ideals $\mathrm{I}_{1}$ and $\mathrm{I}_{2}$.

\section{Relative weak convergence in semifinite real $\mathrm{W}^{*}$-algebras}

In this section, we study the relative weak (RW) convergence in a real Hilbert space. We first recall that the elements of the two-sided closed ideal $I$ generated by the projections which are finite relative to a real $\mathrm{W}^{*}$-algebra $(M, \alpha)$ are called compact operators of $(M, \alpha)$.

Let $H_{r}$ be a real Hilbert space with $H_{r}+i H_{r}=H$. A sequence $\left\{\xi_{n}\right\} \subset H_{r}$ (or $\subset H$ ) is called weakly converging to $\xi$, if for every projection $P$ which is finite relative to $B\left(H_{r}\right)$ (respectively, $\left.B(H)\right), P \xi_{n}$ converges strongly to $P \xi\left(P \xi_{n} \stackrel{S}{\longrightarrow} P \xi\right)$. This suggests the following generalization:

Definition 2 Let $(M, \alpha)$ be a real W*-algebra in $B\left(H_{r}\right) \subset B(H)=B\left(H_{r}\right)+i B\left(H_{r}\right)$. We say that a sequence $\left\{\xi_{n}\right\} \in H_{r}$ converges to $\xi$ weakly relative to $(M, \alpha)$ and briefly say $(\mathrm{RW})_{r}$ converges or $\xi_{n} \stackrel{(R W)_{r}}{\longrightarrow} \xi$, if

1. $\left\|\xi_{n}\right\|$ is bounded;

2. for every projection $P \in(M, \alpha)$ which is finite relative to $(M, \alpha)$, the sequence $\left\{P \xi_{n}\right\}$ converges strongly to the element $P \xi$, i.e. $P \xi_{n} \stackrel{S}{\longrightarrow} P \xi$.

Note that a weakly convergent sequence is necessarily bounded, but following the Example 2 of [7], it is easy to construct an example, in which a unbounded sequence satisfies the second condition of Definition 1.

Example Let $H$ and $K$ be infinite-dimensional separable real Hilbert spaces with orthonormal bases $\left\{\eta_{n}\right\},\left\{\gamma_{n}\right\}$ respectively. We put $R=B(H) \otimes \mathbb{R} \mathbf{I}_{K}$ and $\xi_{n}=$ $\sum_{i=n}^{2 n} \eta_{i} \otimes \gamma_{i}$. Since $\left\|\xi_{n}\right\|^{2}=\sum_{i=n}^{2 n}\left\|\eta_{i}\right\|^{2}\left\|\gamma_{i}\right\|^{2}=n$, it is an unbounded sequence. Let $P$ be a finite protection of $R$. Then there is a finite projection $P_{0}$ in $B(H)$ such that $P=P_{0} \otimes \mathbf{I}_{K}$. Without loss of generality we may assume that it is one-dimensional, i.e., that $P_{0}=<\cdot, \xi>\xi$, for an element $\xi \in H$ with $\|\xi\|=1$. Then

$$
\begin{aligned}
\left\|P \xi_{n}\right\|^{2} & =\left(\sum_{i=n}^{2 n} P_{0} \eta_{i} \otimes \gamma_{i}, \sum_{j=n}^{2 n} P_{0} \eta_{j} \otimes \gamma_{j}\right) \\
& =\left(\sum_{i=n}^{2 n}<\eta_{i}, \xi>\xi \otimes \gamma_{i}, \sum_{j=n}^{2 n}<\eta_{j}, \xi>\xi \otimes \gamma_{j}\right) \\
& =\sum_{i, j=n}^{2 n}\left(<\eta_{i}, \xi>\xi,<\eta_{j}, \xi>\xi\right)_{H} \cdot\left(\gamma_{i}, \gamma_{j}\right)_{K}=\sum_{k=n}^{2 n}\left|<\eta_{k}, \xi>\right|^{2} .
\end{aligned}
$$


Since the series $\sum_{k=1}^{\infty}\left|<\eta_{k}, \xi>\right|^{2}$ converges, the sequence $\sum_{k=n}^{2 n}\left|<\eta_{k}, \xi>\right|^{2}$ converges to 0 , when $n \rightarrow \infty$. Hence $\left\|P \xi_{n}\right\| \rightarrow 0$. Therefore $P \xi_{n} \stackrel{S}{\longrightarrow} P \xi=0$, but the sequence $\left\{\xi_{n}\right\}$ is unbounded. Moreover it is easy to show that the sequence $\left\{\eta_{n} \otimes \gamma\right\}$ $(R W)_{r}$ converges to 0 , but it does not converge strongly to 0 , and the sequence $\left\{\xi \otimes \gamma_{n}\right\}$ converges weakly to 0 , but does not $(R W)_{r}$ converge to 0 .

It is easy to see that the following assertions are true.

\section{Lemma 4}

$$
\xi_{n} \stackrel{R W}{\longrightarrow} \xi \Longleftrightarrow \xi_{n} \stackrel{(R W)_{r}}{\longrightarrow} \xi
$$

Here $\xi_{n} \stackrel{R W}{\longrightarrow} \xi$ means that $\left\|\xi_{n}\right\|$ is bounded and $P \xi_{n} \stackrel{S}{\longrightarrow} P \xi$, for every projection $P \in M$ (see [7, Definition 1]).

Proof The proof of implication " $\Rightarrow$ " is obvious. Let's prove the implication " $\Leftarrow$ ". Assume, that there is some projection $p \in M$ with $\left\|p \xi_{n}\right\| \not \rightarrow 0$. Since the projections $p$ and $\alpha(p)$ are equivalent we have $\left\|\alpha(p) \xi_{n}\right\| \not \nrightarrow 0$ (see [1]). It is easy to see that $a=p+\alpha(p) \in(M, \alpha)$ and $a \geq p$ (because $\alpha(p) \geq 0)$, therefore $\left\|a \xi_{n}\right\| \not \rightarrow 0$. Then exists a spectral projection $e \in(M, \alpha)$ of an element $a$ with $\left\|e \xi_{n}\right\| \not \rightarrow 0$. It is a contradiction with the assumption.

The following theorem is a generalization of Hibert's characterization of the compact operators.

Theorem 5 (Theorem 7, [7]) An element A is compact in $M$ iff it maps (RW) converging sequences into strongly converging ones.

We have the following real analogue of the above characterization.

Theorem 6 An element $A$ is compact in $(M, \alpha)$ iff it maps $(R W)_{r}$ converging sequences into strongly converging ones.

Proof Firstly, let us prove the sufficiency. Let $I$ be the two-sided closed (pure real) ideal generated by the projections which are finite relative to a real $\mathrm{W}^{*}$-algebra $(M, \alpha)$ and put $J=I+i I$. As it was noticed above $J$ is the two-sided closed (complex) ideal, generated by the projections which are finite relative to a $\mathrm{W}^{*}$-algebra $M$. If we put

$$
I_{1}=\left\{A \in(M, \alpha): A \xi_{n} \stackrel{S}{\rightarrow} 0, \text { for any sequence }\left\{\xi_{n}\right\} \subset H_{r} \text { with } \xi_{n} \stackrel{(R W)_{r}}{\longrightarrow} 0\right\}
$$

then by (1) for

$$
J_{1}=\left\{A \in M: A \xi_{n} \stackrel{S}{\rightarrow} 0 \text {, for any sequence }\left\{x_{n}\right\} \subset H \text { with } \xi_{n} \stackrel{(R W)}{\longrightarrow} 0\right\},
$$

we obtain $I_{1} \subset J_{1}=J=I+i I$. Here the equality $J_{1}=J$ is valid by Theorem 5 . Since $I_{1} \subset(M, \alpha)$, we obtain $I_{1} \subset I$. Therefore $A \in I$, i.e. $A$ is compact relatively to $(M, \alpha)$ 
Now we shall prove the necessity. It suffices to show that $I \subset I_{1}$. Suppose that $K \in I$ and $\xi_{n} \stackrel{(R W)_{r}}{\longrightarrow} \xi$. Without loss of generality we can assume that $\xi=\theta$. We repeat step by step the scheme of proof of Theorem 1.3 [8], to obtain the real analogue of the generalized Rellich condition for $K$, i.e., for every $\lambda>0$ there is a projection $P$ of $(M, \alpha)$ such that $\|K P\| \leq \lambda$ and $\mathbf{I}-P$ is finite. From $\xi_{n} \stackrel{(R W)_{r}}{\longrightarrow} 0$, by definition, we obtain $(\mathbf{I}-P) \xi_{n} \stackrel{S}{\longrightarrow} 0$, and hence $K(\mathbf{I}-P) \xi_{n} \stackrel{S}{\longrightarrow} 0$. Since $\|K-K(\mathbf{I}-P)\|=$ $\|K P\| \leq \lambda$ and $\left\|\xi_{n}\right\|$ is bounded by definition, we have $K \xi_{n} \stackrel{S}{\longrightarrow} 0$. Thus, we have shown that for any $K \in I$ the condition $\xi_{n} \stackrel{(R W)_{r}}{\longrightarrow} \xi$ implies $K \xi_{n} \stackrel{S}{\longrightarrow} K \xi$. Hence $K \in I_{1}$, and therefore $I_{1} \subset I$.

Theorem 6 shows that Hibert's characterization of the compact operators remains valid in semifinite real $\mathrm{W}^{*}$-algebras.

Acknowledgments The second and the third named authors would like to acknowledge the hospitality of the "Institut für Angewandte Mathematik", Universität Bonn. This work is supported in part by the DFG AL 214/36-1 project (Germany).

Open Access This article is distributed under the terms of the Creative Commons Attribution License which permits any use, distribution, and reproduction in any medium, provided the original author(s) and the source are credited.

\section{References}

1. Ayupov, Sh.A, Rakhimov, A.A., Usmanov, ShM: Jordan, Real and Lie Structures in Operator Algebras. Kluw. Acad. Pub., MAIA 418, 235 (1997)

2. Ayupov, Sh.A., Rakhimov, A.A.: Real W*-algebras, Actions of groups and index theory for real factors. VDM publishing house ltd. Beau-Bassin, Mauritius. ISBN 978-3-639-29066-0, p. 138 (2010)

3. Breuer, M.: Fredholm theories in von Neumann algebras I. Math. Ann. 178, 243-254 (1968)

4. Connes, A.: Une classification des Facteurs de type III. Ann. Sc. Ec. Norm. Sup. 6, 133-252 (1973)

5. Giordano, T.: Antiautomorphismes involutifs des facteurs de von Neumann injectifs. Thèse, Universite de Neuchâtel, Switzerland, p 106 (1981)

6. Halpern, H., Kaftal, V.: Compact operators in type $\mathrm{III}_{\lambda}$ and $\mathrm{III}_{0}$ factors. Math. Ann. 273, 251-270 (1986)

7. Kaftal, V.: Relative weak convergence in semifinite von Neumann algebras. Am. Math. Soc. 84(1), 89-94 (1982)

8. Kaftal, V.: On the theory of compact operators in von Neumann algebras. I. Indiana Univ. Math. J. 26, 447-457 (1977)

9. Rakhimov, A.A.: Injective real W*-factors of type $\mathrm{III}_{\lambda}, 0<\lambda<1$. Funct. Anal. Appl. 3, 41-44 (1997)

10. Rakhimov, A.A., Katz, A.A., Dadakhodjaev, R.A.: Real compact operators in factors of type I, II, and $\mathrm{III}_{\lambda}, 0<\lambda<1$. Mo. J. Math. Sci. 18(1) (2006)

11. Sonis, M.G.: On a class of operators in von Neumann algebras with segal measures. Math. USSR Sb. 13, 344-359 (1971)

12. Stacey, P.J.: Real structure in $\sigma$-finite factors of Type $\mathrm{III}_{\lambda}$, where $0<\lambda<1$. Proc. Lond. Math. Soc. 47, 275-284 (1983)

13. Usmanov, Sh.M.: Classification of real $\mathrm{W}^{*}$-factors of type $\mathrm{III}_{\lambda}, 0<\lambda<1$. Preprint: $8082-84$, deposed at VINITI (1984)

14. Usmanov, ShM: Classification of real $\mathrm{W}^{*}$-factors of type $\mathrm{III}_{\lambda}, 0 \leq \lambda<1$. Funkt. Anal. i Ego Pril. 19(3), 94-95 (1985)

15. Usmanov, ShM: Operator-valued weights on real $\mathrm{W}^{*}$-algebras and reversible JW-algebras. Sbornik Math. 190(10), 105-122 (1999)

16. Usmanov, ShM: Conditional expectations on real $\mathrm{W}^{*}$-algebras and JW-algebras. Izv. Vyssh. Uchebn. Zaved. Mat. 7, 43-47 (2001) 